\title{
TRATAMIENTO CUARENTENARIO CONTRA LA MOSCA MEDITERRÁNEA Ceratitis capitata WIEDEMANN EN MANGOS VARIEDAD HADEN CON IRRADIACIÓN GAMMA $\left(\mathrm{CO}^{60}\right)$
}

\author{
Quarantine treatment against mediterranean fruit fly (Ceratitis capitata Wiedemann) in \\ mangoes haden variety (Mangifera indica) with gamma irradiation $\left(\mathrm{Co}^{60}\right)$
}

María E. Peña C. ${ }^{2}$, Rafael Guillen E. ${ }^{1}$, Gladys C. Arias A. ${ }^{2}$

${ }^{1}$ Servicio Nacional de Sanidad Agraria - SENASA. ${ }^{2}$ Laboratorio de Bromatología Facultad de Farmacia y Bioquímica Universidad Nacional Mayor de San Marcos

\section{RESUMEN}

Las moscas de la fruta, en especial la Ceratitis capitata Wiedemann, son una de las plagas más dañinas del mundo, atacan a más de 250 especies frutícolas y hortalizas, constituyendo la principal restricción fitosanitaria para su exportación. La investigación fue realizada en el Centro de Producción de Moscas de la Fruta Estéril del SENASA y el laboratorio de la Planta de Irradiación Multiuso (PIMU), supervisada por un experto del Organismo Internacional de Energía Atómica (OIEA). La investigación consistió en dos etapas: 1) Determinar la dosis mínima efectiva de irradiación a la cual no haya emergencia de mosca adulta en mango (prueba de bioensayo) y 2) Evaluar 30 ooo individuos a la dosis mínima efectiva, que proporciona datos estadísticamente confiables (prueba confirmatoria); confirmándose que a o,1 kGy no hay emergencia. De la evaluación química y organoléptica, se determinó que no existe diferencia significativa frente a los mangos que no fueron tratados.

Palabras clave: Ceratitis capitata Wiedemamm, mango, Haden, irradiación, moscas de la fruta.

\section{SUMMARY}

Tephritidae fruit flies are among the most damaging pests of fruit crops worldwide Ceratitis capitata Wiedemann, for instance, can infest more than 250 fruit and vegetable crop species, and constitutes the main phytosanitary restrict for export purposes. The investigation was carried out in a SENASA's Production Center of Sterile Fruit Flies and PIMU's laboratory; it was supervised by an OIEA's irradiation expert. This research comprised two stages: 1) Determine the minimum irradiation dose to avoid emergence of fruit fly adults (bioassay test), and 2) Evaluate 30 ooo individuals having been exposed to the minimum effective dose to provide statistically reliable data (confirmatory test). It was confirmed that no adult emergence occurred at o, $1 \mathrm{kGy}$, and no significant differences were found between treated and non-treated mangoes when both chemical and organoleptic tests were applied.

Keywords: Ceratitis capitata Wiedemamm, handle, Haden, irradiation, flies of the fruit.

\section{INTRODUCCIÓN}

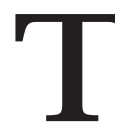

ratamientos de cuarentena son usados en hospederos de moscas de la fruta para prevenir la introducción de estas especies y evitar que puedan diseminarse en áreas donde no se encuentren naturalmente. Los tratamientos aprobados para este fin incluyen: fumigación con bromuro de metilo, inmersión en agua caliente, uso de vapor caliente, aire caliente y el almacenamiento en frío; atmósfera modificada con oxígeno bajo o niveles altos de anhídrido carbónico, y certificación de zonas libres de mosca de la fruta que no requieren el tratamiento.

La irradiación ha mostrado ser una alternativa viable como un tratamiento de cuarentena para eliminar esta plaga. Desde 1999 en nuestro país se realizan estudios para determinar la viabilidad de usar la irradiación como un tratamiento de cuarentena para frutas y hortalizas frescas sujetas a las restricciones fitosanitarias para el comercio ${ }^{(1-3)}$.

El objetivo del presente trabajo fue determinar la dosis mínima efectiva para larvas del tercer estadio de Ceratitis capitata Wiedemann, que asegure la no emergencia de adultos en mangos y evaluar los efectos de irradiación sobre la calidad de la fruta, mediante análisis sensorial y químico para validar el uso de la irradiación como tratamiento de cuarentena para la exportación de frutas frescas. 


\section{MATERIALES Y MÉTODOS}

\section{Pruebas de bioensayo}

a) Selección de frutas. Se seleccionaron mangos en estado de madurez verde-sazón, cuya cáscara tuviera una apariencia verde con chapa roja, de consistencia firme, en perfecto estado de desarrollo fisiológico, libre de infestación y con un peso promedio de 300 a 400 g por mango.

b) Infestación de frutos. Se realizó en condiciones de laboratorio, mediante el método de oviposición natural ${ }^{(4)}$. Los mangos debían tener un rango de $\mathrm{pH}$ de 3,7 a 4,o pues en ese estado son más susceptibles a la infestación de la mosca y esta puede depositar sus huevos en el fruto.

c) Desarrollo larvario. $\mathrm{Al}$ término de la infestación, los frutos se retiraron de las jaulas y se colocaron en bandejas de plástico con un sustrato de pupación (aserrín) para la recuperación larval; éstas se mantuvieron en condiciones ambientales de temperatura $26{ }^{\circ} \mathrm{C} \pm 2$ y una humedad relativa de 70 a $75 \%$ con la finalidad de que se realice el desarrollo larvario.

d) Proceso de irradiación. Cuando las larvas de los mangos infestados se encontraron en el tercer estadio, estado biológico más radioresistente, se procedió a la irradiación de la fruta. El proceso se realizó después de 8 a 9 días de la infestación, previa disección de un mango para verificar el estadio de las larvas, colocándose los mangos en cajas de cartón tipo exportación, simulando el empaque final de los frutos.

Para la irradiación, cada lote de mangos fue separado en dos grupos, 50\% para el control y 50\% para la prueba de bioensayo. Los mangos fueron irradiados por etapas: el primer lote a o,15 kGy, luego se analizó no encontrándose emergencia de mosca adulta, por lo que se bajó la dosis a o,12 y así sucesivamente hasta encontrar emergencia de mosca adulta, entonces se tomó la dosis inmediata superior y se procedió a la prueba confirmatoria.

Tabla 1. Irradiación de moscas

\begin{tabular}{cc}
\hline $\begin{array}{c}\text { Dosis } \\
\text { kGy }\end{array}$ & $\begin{array}{c}\text { Tiempo de exposición } \\
\text { (Minutos) }\end{array}$ \\
\hline 0,15 & 53,05 \\
0,12 & 42,44 \\
0,10 & 35,37 \\
0,075 & 26,52 \\
\hline
\end{tabular}

e) Acondicionamiento para pupación. Terminada la irradiación, los mangos fueron colocados sobre una base de aserrín en bandejas de plástico para la pupación de las larvas que abandonan el fruto. Las condiciones ambientales a las que se mantuvieron fueron: temperatura $24^{\circ} \mathrm{C}$, humedad relativa 60 a $65 \%$ y sin iluminación para evitar el salto de las larvas.

f) Tamizado. Al sexto día de pupación se realizó la separación de la pupa del aserrín con la ayuda de un tamiz y se procedió a su colecta. Se midió el volumen de pupas colectadas y se contaron; el resultado se llevó a una base de datos.

h) Acondicionamiento para emergencia. Las pupas colectadas del lote irradiado se colocaron en jaulas pequeñas para que estas continúen su desarrollo hasta la emergencia de mosca adulta; cada jaula fue codificada para evitar confusión.

\section{Prueba confirmatoria}

Una vez determinada la dosis mínima efectiva se inició la prueba confirmatoria, de acuerdo con el protocolo de investigación para el uso de las radiaciones ionizantes como tratamiento cuarentenario en frutas, Reglamento cuarentenario armonizado para la irradiación de frutas PROYECTO RLA / 5 / 043 - ARCAL XXXIII. El análisis de resultados logrados con el tratamiento de 30 ooo individuos permitirá obtener datos estadísticamente confiables ${ }^{(5)}$.

Para esta prueba se repitió el mismo procedimiento de la prueba de bioensayo, con la diferencia que los lotes se irradiaron a una misma dosis (dosis mínima efectiva); además, cada lote de fruta infestada se dividió en dos grupos: uno de $75 \%$, el cual se trató con dosis mínima efectiva, y el otro de $25 \%$ como testigo.

\section{Evaluación sensorial}

La evaluación sensorial estuvo a cargo de un panel de degustación semientrenado. Los parámetros evaluados fueron: olor, color, sabor y textura.

Se aplicó el análisis Duo-Trío que emplea tres muestras codificadas: dos idénticas y una diferente, donde una de las idénticas es la muestra patrón ${ }^{(6)}$.

Se evaluó presentando al panelista primero la muestra patrón (o,o kGy), luego las otras dos (o,o y o,1 kGy) debidamente codificadas para que proceda a compararlas e identifique cuál de éstas es igual a la del patrón.

Para determinar si existe evidencia estadística entre la muestra patrón y la muestra irradiada, los datos obtenidos a partir de la evaluación sensorial 
Tabla 2. Resultados de la prueba de bioensayo

\begin{tabular}{|c|c|c|c|c|c|c|c|c|}
\hline & \multicolumn{2}{|c|}{ D1 } & \multicolumn{2}{|c|}{ D2 } & \multicolumn{2}{|c|}{ D3 } & \multicolumn{2}{|c|}{ D4 } \\
\hline & Control & $0,15 \mathrm{kGy}$ & Control & 0,12 kGy & Control & $0,10 \mathrm{kGy}$ & Control & 0,075 kGy \\
\hline № de pupas colectadas & 4457 & 2385 & 3044 & 3773 & 3273 & 4009 & 4093 & 4128 \\
\hline № de moscas emergidas & 4132 & 0 & 2820 & 0 & 3021 & 0 & 3800 & 2 \\
\hline$\%$ emergencia & 92,83 & 0 & 92,64 & 0 & 92,3 & 0 & 92,8 & 0,048 \\
\hline
\end{tabular}

Tabla 3. Prueba confirmatoria

\begin{tabular}{|c|c|c|c|c|c|c|}
\hline \multirow{2}{*}{ Tratamiento } & \multicolumn{2}{|c|}{ № pupas colectadas } & \multicolumn{2}{|c|}{ № moscas emergidas } & \multicolumn{2}{|c|}{$\%$ emergencia } \\
\hline & Control & $0,1 \mathrm{kGy}$ & Control & $0,1 \mathrm{kGy}$ & Control & $0,1 \mathrm{kGy}$ \\
\hline $\mathrm{T}_{1}$ & 653 & 715 & 608 & 0 & 93,10 & 0 \\
\hline $\mathrm{T}_{2}$ & 276 & 2613 & 245 & 0 & 88,77 & 0 \\
\hline $\mathrm{T}_{3}$ & 3186 & 9336 & 2944 & 0 & 92,40 & 0 \\
\hline $\mathrm{T}_{4}$ & 1327 & 4212 & 1229 & 0 & 92,53 & 0 \\
\hline $\mathrm{T}_{5}^{4}$ & 183 & 345 & 169 & 0 & 92,54 & 0 \\
\hline $\mathrm{T}_{6}$ & 2835 & 4092 & 2629 & 0 & 92,75 & 0 \\
\hline $\mathrm{T}_{7}$ & 1097 & 3560 & 1017 & 0 & 92,78 & 0 \\
\hline $\mathrm{T}_{8}$ & 2441 & 5745 & 2265 & 0 & 92,78 & 0 \\
\hline $\mathrm{T}_{9}$ & 1205 & 2983 & 1118 & 0 & 92,82 & 0 \\
\hline $\mathrm{T}_{10}$ & 1203 & 1706 & 1120 & 0 & 93,10 & 0 \\
\hline $\mathrm{T}_{11}$ & 255 & 724 & 238 & 0 & 93,33 & 0 \\
\hline Total & 14661 & 36031 & 13582 & & 92,64 & \\
\hline
\end{tabular}

fueron evaluados por la prueba de Chi-Cuadrado $\left(\mathrm{X}^{2}\right)$ aplicada al Duo-Trío ${ }^{(6)}$.

\section{Determinación de $\mathbf{p H}$}

La determinación de $\mathrm{pH}$ de la pulpa de los mangos se realizó con un potenciómetro digital "Cole Parmer" (o5669-20).

\section{Determinación de acidez}

Se calculó a través de un análisis volumétrico con solución patrón de $\mathrm{NaOH}$ o, $1 \mathrm{~N}$, usando fenolftaleína como indicador.

\section{SST ( ${ }^{\circ}$ Brix)}

Se determinó mediante un refractómetro de mano, corregido por la temperatura del jugo.

\section{Diseño experimental}

Para la determinación de: $\mathrm{pH}$, acidez titulable y sólidos solubles totales, se utilizó el diseño completamente al azar (DCA) porque es el que mejor se aplica a este trabajo de investigación, ya que éste es útil para trabajos de laboratorio donde la variación entre las unidades experimentales es muy pequeña. Luego se efectuó la prueba de comparaciones múltiples de Tukey.

\section{RESULTADOS}

De las pruebas de bioensayo, se puede apreciar que el porcentaje de emergencia de mosca adulta para las dosis de 0,15; 0,12 y 0,10 kGy fue de o,o\%; mientras que para los controles se tuvo un porcentaje de emergencia en promedio del 92,64\% (Tabla 2).

En la tabla 2, se puede observar que la emergencia de mosca adulta, para la dosis de o,1o kGy, fue nula, teniendo como base que la infestación fue buena, con un porcentaje promedio de emergencia para el lote control de $92,64 \%$.

Evaluación sensorial. Los resultados de los parámetros de olor, color, sabor y textura se evaluaron con el estadístico Chi-Cuadrado $\left(\mathrm{X}^{2}\right)$ aplicado al DuoTrío, el cual nos demostró que no existe diferencia entre el lote tratado con la dosis mínima efectiva (o,1 kGy) y testigo (o,o kGy).

Análisis estadístico de $\mathbf{p H}$. Se realizó mediante DCA. De acuerdo a la prueba de Tukey no hay diferencias estadísticas entre las dosis de irradiación como se puede apreciar en la tabla 4; el promedio más alto fue de o,10 kGy con 5,4420.

El análisis estadístico de acidez titulable. Se realizó por DCA. De acuerdo a la prueba de Tukey no hay diferencias estadísticas entre las dosis de irradiación como se puede apreciar en la tabla 5, pero hay incremento referente al testigo.

El análisis estadístico de la determinación de sólidos totales, se realizó por el DCA. De acuerdo a la prueba de Tukey (se utilizó $\sqrt{\mathrm{x}+5}$, según Steel y Torre) no hay diferencias estadísticas entre las dosis 
Tabla 4. Prueba de Tukey al 5\% en determinación de $\mathrm{pH}(\mathrm{n}=5)$

\begin{tabular}{lcccc}
\hline \multirow{2}{*}{ Estadísticos } & \multicolumn{4}{c}{ Dosis } \\
\cline { 2 - 5 } & Testigo & $\mathbf{0 , 1 5} \mathbf{~ k G y}$ & $\mathbf{0 , 1 2} \mathbf{~ k G y}$ & $\mathbf{0 , 1 0} \mathbf{~ k G y}$ \\
\hline Subconjunto 1 & 5,1380 & 5,1940 & 5,3280 & 5,4420 \\
\hline
\end{tabular}

Tabla 5. Prueba de Tukey al 5\% en determinación de acidez titulable (n=5)

\begin{tabular}{ccccc}
\hline \multirow{2}{*}{ Estadísticos } & \multicolumn{4}{c}{ Dosis } \\
\cline { 2 - 5 } & Testigo & $\mathbf{0 , 1 0} \mathbf{~ k G y}$ & $\mathbf{0 , 1 2} \mathbf{~ k G y}$ & $\mathbf{0 , 1 5} \mathbf{~ k G y}$ \\
\hline $\begin{array}{l}\text { Subconjunto para } \\
\text { alfa }=0.05\end{array}$ & 0,5940 & 0,7240 & 0,8020 & 0,834 \\
\hline
\end{tabular}

Tabla 6. Prueba de Tukey al 5\% en determinación de sólidos solubles (n=5)

\begin{tabular}{ccccc}
\hline \multirow{2}{*}{ Estadísticos } & \multicolumn{5}{c}{ Dosis } \\
\cline { 2 - 5 } Subconjunto 1 & Testigo & $\mathbf{0 , 1 2} \mathbf{~ k G y}$ & $\mathbf{0 , 1 0} \mathbf{~ k G y}$ & $\mathbf{0 , 1 5} \mathbf{~ k G y}$ \\
\hline \multirow{2}{*}{ Subcon } & 5 & 5 & 5 & 5 \\
& 12,20 & 12,12 & 12,60 & 13,16 \\
\hline
\end{tabular}

Se muestran las medias para los grupos en los subconjuntos homogéneos.

Usa el tamaño muestral de la media armónica $=5,000$

de irradiación, como se puede apreciar en la tabla 6, pero hay incremento referente al testigo.

\section{DISCUSIÓN}

Los valores obtenidos de las pruebas de bioensayo, nosindican quelas condicionesen lasquese mantuvieron las larvas (ambientales y alimentación) fueron las óptimas: o,o\% de emergencia de mosca adulta para las dosis de o,15; o,12 y o,10 kGy, y para los controles un porcentaje de emergencia promedio del $92,64 \%$.

Asimismo, para la dosis de o,075 kGy se obtuvo una emergencia de 2 moscas, lo que nos llevó a realizar la prueba confirmatoria con la dosis inmediata superior (o,10 kGy).

En los resultados obtenidos por Bustos et al., cinco de 106100 larvas del tercer estadio, en mangos irradiados con $150 \mathrm{kGy}(\mathrm{o}, 23 \mathrm{~Gy} / \mathrm{s})$ sobrevivieron al estado adulto. Sin embargo, los cinco sobrevivientes ocurrieron dentro de las primeras 5300 larvas testeadas (prueba de laboratorio), no ocurriendo ninguno en el resto de las más de 100000 larvas irradiadas (Prueba confirmatoria). También, la data de la dosis respuesta de la prueba (prueba de laboratorio) mostró que el nivel de emergencia de adultos permaneció estable entre o,09-0,16\% de 8o a $150 \mathrm{kGy}$, declinando gradualmente conforme se incrementa la dosis. Este tipo de relación entre dosis y respuesta es indicativo de una población mixta donde muchos de los individuos son significativamente más tolerantes a la irradiación que el resto ${ }^{(4)}$.
Para la prueba confirmatoria, por cada muestra de fruta infestada se seleccionó al azar el $75 \%$ y se trató a una dosis de o,1 kGy; el resto de fruta (25\%) se manejó como testigo.

En la tabla 3 se puede observar que la emergencia de mosca adulta para o, $\mathrm{kGy}$ fue nula, teniendo como base que la infestación fue buena, con un porcentaje promedio de emergencia para el lote control de $92,64 \%$. También podemos decir que el resultado obtenido de 36031 pupas no emergidas nos da un dato estadísticamente confiable, lo que indicaría que la dosis de o,10 $\mathrm{kGy}$ es una $\mathrm{LD}_{99}$. Thomas también encontró que $40 \mathrm{kGy}$ aplicados a mangos infestados con larvas de siete días de edad previene la emergencia de adultos ${ }^{(7)}$.

De la evaluación sensorial, los resultados de los parámetros: olor, color, sabor y textura se evaluaron con el estadístico de Chi-Cuadrado $\left(\mathrm{X}^{2}\right)$ aplicado al Duo-Trío, el cual nos demostró que no existe diferencia del lote tratado a la dosis mínima efectiva (o,1 kGy) frente al lote testigo (o.o kGy). La no existencia de diferencia significativa se debe a que la dosis de irradiación es muy baja y si existiese alguna diferencia podría deberse al estado de madurez de los mangos, ya que es variable.

Moy et al. (1971) reportan que mangos Haden pueden tolerar dosis de 100 a $150 \mathrm{krad}(1,00$ a 1,50 kGy) sin presentar diferencias en la cualidades organolépticas.

Estudios realizados por Thomas (1986) determinaron que la irradiación a dosis de 25 a 100 
krad (o,25 a 1,oo kGy) no dañó la pulpa de mangos Haden en ningún estado de madurez, para lo cual evaluó las características sensoriales y sus propiedades físicas y químicas ${ }^{(7)}$.

El análisis estadístico de acidez titulable, se realizó por DCA, de acuerdo a la prueba de Tukey no hay diferencias estadísticas entre las dosis de irradiación como se puede apreciar en la tabla 5, pero hay incremento referente al testigo. Quispe (1988) concluye que no hay variación en los porcentajes de acidez de mangos Haden irradiados hasta dosis de o,75 kGy, siempre y cuando sean almacenados a $10^{\circ} \mathrm{C}$ de temperatura ${ }^{(8-10)}$.

\section{CONCLUSIONES}

Los valores hallados en las pruebas de Bioensayo con irradiación gamma $\left(\mathrm{Co}^{60}\right)$ en mangos infestados con larvas en tercer estadio, que recibieron dosis de o,10 kGy, indican que esta cantidad de radiación previene la emergencia de moscas adultas de Ceratitis capitata Wiedemann, pese a que la dosis está por debajo de lo recomendado para causar mortandad larvaria.

De la evaluación sensorial de las pruebas de olor, sabor, color y textura en mangos Haden, se determinó que no existe diferencia significativa a un nivel de confianza del $99,95 \%$ a la dosis mínima efectiva (o,1 kGy).

El pH, acidéz titulable, sólidos solubles totales y ${ }^{\circ}$ Brix de los mangos irradiados, en la Prueba de Tukey al $5 \%$, a diferentes dosis de irradiación (o,15; 0,12 y 0,10 KGy).

\section{REFERENCIAS BIBLIOGRÁFICAS}

1. FAO/AIEA. Training manual on food irradiation, technology and techniques. STI/DOC/10/114/2. Viena, 1982.

2. FAO/AIEA/WHO. A report of the working group convened by ICGFI. Irradiatión as a quarantine treatment of fresh fruits and vegetables. ICGFI.DOC № 17 p 26-30. Viena-Austria 1994.

3. Toledo A, Enkerlin H, Bustos M. Irradiación gamma $\left(\mathrm{Co}^{60}\right)$ en frutas como tratamiento cuarentenario. En: VI curso internacional sobre moscas de la fruta. Programa Moscamed. Chiapas, 1991. p 77-85.

4. Bustos ME. Aplicación de la irradiación para la desinfestación de Mango Kent [Tesis de Maestría], Escuela Nacional de Fruticultura. Universidad de Chapingo. México DF, 1987.

5. ARCAL. Reglamento cuarentenario armonizado para la irradiación de frutas - PROYECTO RLA/5/o43 (ARCAL XXXIII). Viena, 1999.

6. Ureña M. Evaluación sensorial de los alimentos. Aplicación didáctica. Universidad Nacional Agraria de La Molina. Lima, 1999.

7. Thomas P. Radiation preservation of foods of plants origin. III. Tropical fruits: bananas, mangoes and papayas. Crit Rev Food Sci Nutr 1986; 23(2):147-205.

8. Quispe MF. Estudio del efecto de la irradiación gamma en la conservación del mango, de la variedad Haden, almacenado en refrigeración. [Tesis para obtener el título de Ingeniero en Industrias Alimentarias]. Universidad Nacional Agraria de La Molina. Lima, 1988.

9. Muñoz B, Sanchez V, Uzcátegui A, Vaca F. Preservación de alimentos por irradiación. Escuela Politécnica Nacional. Quito, 1985.

10. World Health Organization. Food irradiación. A technique for preserving and improving the safety of food. Geneva. 1988: 84 .

Manuscrito recibido el: 15/11/2010

Aceptado para su publicación el: 01/02/2011

\section{Correspondencia:}

Nombre: Gladys Arias Arroyo

Dirección: Jr. Puno 1002, Lima 1 - Perú.

e-mail: ariasarroyo@gmail.com 